Khazhieva I. S., Glukhareva T. V.,

Morzherin Yu. Yu.

Y-Synthesis, Ltd, 60 Lunacharskogo street, 620027 Ekaterinburg Phone: +7(343) 375-48-18; E-mail:his_inna@mail.ru

\title{
Reaction of 4-acetyl-1,2,3-triazol-5-olate with hydrazine derivatives
}

1,2,3-triazoles attract attention because of thein ability to ring open leading to a-diazoimin and intramolecular rearrangements to form various heterocyclic systems. Previously, the interaction of the $1 H$-1,2,3-triazole-5-ol sodium salt with amine hydrochloride resulting in the formation of isomeric 1,2,3-triazoles has been studied. In this paper, we present a study of the interaction of 4-acetyl-1,2,3-triazole-5-ol sodium salt with hydrazine derivatives. As result, 5-methyl-1,2,3-triazol-4-phenylcarboxamide-phenylamide and bis 5,5'-dimethyl-[1,1']bi $[[1,2,3]$ triazolyl]-4,4'-dicarboxylic acid derivatives were synthesized.

(C) Khazhieva I. S., Glukhareva T. V., Morzherin Yu. Yu., 2015

\section{Introduction}

Among 1,2,3-triazoles there are some compounds which exhibit various biological activities (antibacterial, antitumoral, anti-fungal ${ }^{1}$, immunosuppressive $^{2}$ ), and various technical properties. Currently, the search continues for new 1,2,3-triazole derivatives which have biological activity, and, consequently, there is an increase in number of publications dedicated to 1,2,3-triazoles.

Furthermore, 1,2,3-triazoles exhibit<smiles>[R]N=C([R])C([Y2])=N</smiles>

interesting chemical properties, such as ring-chain isomerisation, rearrangement and transformation of the ring. This is determined by the ability of 1,2,3-triazoles to ring opening with the formation of $\alpha$-diazoimine.

We have previously shown that reaction of sodium 4-acetyl-1,2,3-triazolate 1 with amine hydrochlorides proceeds with the formation of 5-methyl-1,2,3-triazol4-carboxamides $2^{3}$.

In this study, we investigated reaction of sodium 4-acetyl-1,2,3-triazolate 1 with hydrazine derivatives.

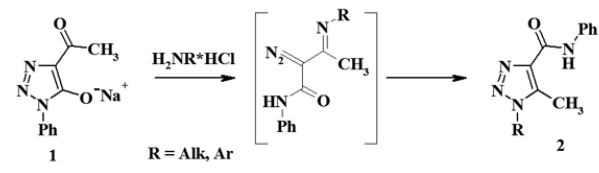




\section{Results and Discussion}

It was shown that the reaction of sodium 1,2,3-triazolate 1 with equimolar amount of hydrazine hydrochlorides $3 \mathrm{a}-\mathrm{g}$ leads to 1-amino-5-methyl-1,2,3-triazol4-yl-carboxamides, 4 a-g .

However, bistriazole 5 was isolated using unsubstituted hydrazine in the re-

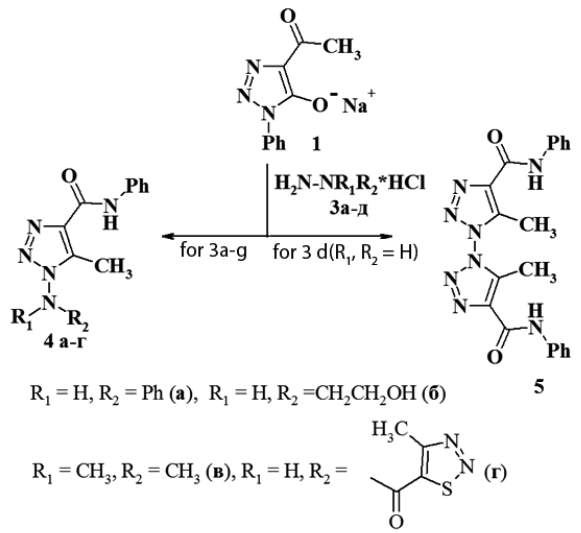

action. In this case, one molecule of hy- drazine reacts with two molecules of triazolate, accompanied by rearrangement of two triazole rings. Perhaps, this course of the reaction is due to slow dissolving of hydrazine hydrochloride in ethanol.

1-Amino-1,2,3-triazol 6 was prepared in the reaction of 2-diazoacetoacetanilid hydrazine hydrate 7 obtained after acidification of sodium 1,2,3-triazolate aqueous solution.

Thus we obtained previously un-

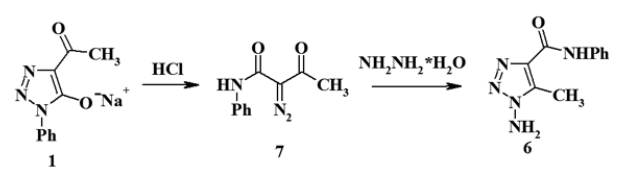

described phenylamide derivatives 5-methyl-1- (R-amino) -1 H [1,2,3] triazole-4-carboxylic acid and bis-phenylamid-5,5'-dimethyl-[1, 1'] bi [[1,2,3] triazolyl] -4,4'-dicarboxylic acid.

\section{The experimental part}

Reaction monitoring and the individuality of the synthesized compounds was performed by TLC plates SolufolUV 254 in the system: chloroform (Visualisation under UV lamp). IR spectra were recorded on a spectrophotometer Bruker Alpha. NMR ${ }^{1} \mathrm{H}$ and ${ }^{13} \mathrm{C}$ spectra were recorded on a spectrometer Bruker AvanceII (400 and $100 \mathrm{MHz}$, respectively), internal standard is TMS, in the Laboratory of comprehensive research and evaluation of organic materials at CCU UFU. Mass spectres were recorded on a spectrometer
MAT 11 (EI, $70 \mathrm{eV}$ ). Melting points were measured using art StuartSMP3 apparatus and were not corrected. Elemental analysis was performed on a CHNS-analyzer PE 2400 SeriesII.

Methods for preparation of $4 \mathrm{a}-\mathrm{g}$ and 5

(1.43 mmol) hydrazine hydrochloride 3 a-d is added to an alcohol solution of sodium triazolate $10.29 \mathrm{~g}(1.3 \mathrm{mmol})$. The reaction mixture was heated for 24 hours. After cooling, the precipitation was filtered off, washed with ethanol and dried.

\section{Phenylamide 5-methyl-1-phenylamino-1H-[1,2,3] triazole-4-carboxylic acid (4a)}

White crystalline solid $0.36 \mathrm{~g} \quad-\mathrm{NH}), 10,39(1 \mathrm{H}, \mathrm{c},-\mathrm{NH}), 7,85(2 \mathrm{H}, \mathrm{d}, \mathrm{J}$ (94.74\%). $\left.\mathrm{Tm}=147.3{ }^{\circ} \mathrm{C} . \mathrm{NMR}{ }^{1} \mathrm{H}: \quad=8,4, \mathrm{o}-\mathrm{Ar}-\mathrm{H}\right), 7,33(2 \mathrm{H}, \mathrm{dd}, \mathrm{J}=7,5 ; \mathrm{J}=$ (DMSO-D, d, ppm, J / Hz): $10.41(1 \mathrm{H}, \mathrm{c}, \quad 8,4, \mathrm{~m}-\mathrm{Ar}-\mathrm{H}), 7,23(2 \mathrm{H}, \mathrm{dd}, \mathrm{J}=7,5 ; \mathrm{J}=$ 
8,4, m-Ar-H), 7,09 (1H, dd, J = 8,4; J = 7,5, p-Ar-H), 6,92 (1H, dd, $\mathrm{J}^{1}=8,4 ; \mathrm{J}^{2}=$ $7,5, \mathrm{p}-\mathrm{Ar}-\mathrm{H}), 6,51(2 \mathrm{H}, \mathrm{d}, \mathrm{J}=8,4$, a-Ar$\mathrm{H}), 2,47\left(3 \mathrm{H}, \mathrm{s}, \mathrm{CH}_{3}\right)$. NMR ${ }^{13} \mathrm{C}$ specter: (DMSO-D6,d, ppm): 159.61, 146.76,
$139.02,138.59,137.67,124.16,121.82$, 120.88, 11337 8.7. Found, \%: C 65,50, H $5,17, \mathrm{~N} 23,91$. Calculated for $\mathrm{C}_{16} \mathrm{H}_{15} \mathrm{~N}_{5} \mathrm{O}$, $\%$ : C 65,52, H 5,15, N 23,88, O 5,45.

\section{Phenylamide 5-methyl-1-(2-hydroxy-ethylamine)-1H-[1,2,3]triazole-4- carboxylic acid (4b)}

Dark yellow oil, $0.36 \mathrm{~g}(89.45 \%)$. NMR ${ }^{1} \mathrm{H}$ : (DMSO-D6, d, ppm, J / Hz): $9.99(1 \mathrm{H}, \mathrm{c},-\mathrm{NH}), 7,82(2 \mathrm{H}, \mathrm{d}, \mathrm{J}=7,6$ o-Ar- H), 7,31 (2H, dd, $\mathrm{J}=7,6 ; \mathrm{J}=8,3$, m-Ar-H), 7,20 (1H, t., J = 5,4, -NH ), 7.05 (1H, dd., $\mathrm{J}=7,4 ; \mathrm{J}=7,4$, p-Ar-H),
4,06 (1H, bs, -OH) 3,56 (2H, b.t., $\mathrm{J}=5,5$, $\left.\mathrm{CH}_{2}\right), 3.26\left(1 \mathrm{H}, \mathrm{dd}, \mathrm{J}=5,5, \mathrm{~J}=5,4, \mathrm{CH}_{2}\right)$ 2,56 $\left(3 \mathrm{H}, \mathrm{s}, \mathrm{CH}_{3}\right)$. Found, \%: $\mathrm{C} 55,15 \mathrm{H}$ $5,80 \mathrm{~N} 26,80$. Calculated for $\mathrm{C}_{12} \mathrm{H}_{15} \mathrm{~N}_{5} \mathrm{O}_{2}$, \%: C 55,16, H 5,79, N 26,80, O 12,25.

\section{Phenylamide 5-methyl-1-dimethylamino-1H-[1,2,3] triazole-4-carboxylic acid (4c)}

Dark yellow oil, 0.34 g (92.73\%). NMR ${ }^{1} \mathrm{H}$ : (DMSO-D6, d, ppm, J / Hz): 10.06 (1H, c, -NH), 7,82 (2H, d, J = 7,6, o-Ar $-\mathrm{H}), 7,29$ (2H, dd, $J=7,6 ; J=7,4$, $\mathrm{m}-\mathrm{Ar}-\mathrm{H}), 7,05$ (1H, dd, $\mathrm{J}=7,4 ; \mathrm{J}=7,4$,
p-Ar-H), 3,02 (3H, s, $\left.\mathrm{CH}_{3}\right) 3,06(3 \mathrm{H}, \mathrm{s}$, $\left.\mathrm{CH}_{3}\right)$ 2,54 (3H, s, $\left.\mathrm{CH}_{3}\right)$. Found, \%: C $55,77 \mathrm{H} 6,15 \mathrm{~N}$ 28,57. Calculated for $\mathrm{C}_{12} \mathrm{H}_{15} \mathrm{~N}_{5} \mathrm{O}, \%$ : C 58,76, H 6,16, N 28,55, O $6,52 . \%$

\section{(5-methyl-4-phenylcarboxamide - $[1,2,3]$ triazole-1-yl)-amide 4-methyl}

\section{$[1,2,3]$ thiadiazole-5-carboxylic acid (4a)}

White crystalline solid of $0.52 \mathrm{~g}$ $(82.35 \%) . \mathrm{Tm}=175,6^{\circ} \mathrm{C} . \mathrm{NMR}{ }^{1} \mathrm{H}$ : (DMSO-D6, d, ppm, J / Hz): $13.23(1 \mathrm{H}, \mathrm{c}$, -NH), 10,28 (1H, c, -NH), 7,84 (2H , d, $\mathrm{J}=7,8, \mathrm{o}-\mathrm{Ar}-\mathrm{H}), 7,30(2 \mathrm{H}, \mathrm{dd}, \mathrm{J}=7,8$;
$\mathrm{J}=7,9, \mathrm{~m}-\mathrm{Ar}-\mathrm{H}), 7,07$ (1H, t., $\mathrm{J}=7,9$; $\mathrm{J}=7,9, \mathrm{p}-\mathrm{Ar}-\mathrm{H}), 2,96\left(3 \mathrm{H}, \mathrm{s}, \mathrm{CH}_{3}\right) 2,55$ $\left(3 \mathrm{H}, \mathrm{s}, \mathrm{CH}_{3}\right)$. Found, \%: C 48,98 H 3,81 $\mathrm{N} 28,53$. Calculated for $\mathrm{C}_{14} \mathrm{H}_{13} \mathrm{~N}_{7} \mathrm{O}_{2} \mathrm{~S}, \%$ : C 48,97; H 3,82, N 28,55; O 9,32, S 9,34.

\section{Bis-phenylamide 5,5'-dimethyl-[1,1 '] bi [[1,2,3] triazolyl] $-4,4^{\prime}$-dicarboxylic acid (5)}

White crystalline solid $0.30 \mathrm{~g}$ (84.35\%). $\mathrm{Tm}=217^{\circ} \mathrm{C}\left(180^{\circ} \mathrm{C}\right.$ years $)$. NMR ${ }^{1} \mathrm{H}$ : (DMSO-D6, d, ppm, J / Hz): $9.02(2 \mathrm{H}, \mathrm{c},-\mathrm{NH}), 7,74(4 \mathrm{H}, \mathrm{d}, \mathrm{o}-\mathrm{Ar}-\mathrm{H}$ $\mathrm{J}=8,0), 7.44(4 \mathrm{H}, \mathrm{dd}, \mathrm{J}=7,6 ; \mathrm{J}=8,4 \mathrm{~m}-$ Ar-H), 7,23 (2H, dd, J=7,2, p-Ar -H). IR, $\left(\mathrm{v}, \mathrm{cm}^{-1}\right) 3308,98(-\mathrm{NH}), 1666,04(\mathrm{C}=\mathrm{O})$. Mass specter (EI, 70 eV), m / z (Ires (\%)): $[\mathrm{M}]+402$ (9.14). Found, \%: C 59,71 H
4,47 N 18,04. Calculated for $\mathrm{C}_{20} \mathrm{H}_{8} \mathrm{~N}_{8} \mathrm{O}_{2}$, \%: C 59,70; H 4,48 N 27,86; O 7,96.

Sinthesis of 6

Hydrazine hydrate $0.07 \mathrm{~g}(1.43 \mathrm{mmol})$ is added to an alcohol solution of 2- diazoacetanilid $70.26 \mathrm{~g}(1.3 \mathrm{mmol})$. The reaction mixture was refluxed for a day. After cooling the priecipitate was filtered, washed with ethanol, and dried. 
Phenylamid 5-methyl-1-amino-1H-[1,2,3] triazole-4-carboxylic acid (4c)

White crystalline solid of $0.32 \mathrm{~g}$ (2H, c., -NH2), 2,54 (1H, c, -3). Mass $(83.39 \%) . \mathrm{Tm}=199^{\circ} \mathrm{C}\left(120^{\circ} \mathrm{C}\right.$ years). $\quad$ spectrum $3(\mathrm{EI}, 70 \mathrm{eV}), \mathrm{m} / \mathrm{z}$ (Irel (\%)): NMR 'H: (DMSO-D6, d, ppm, J / Hz): [M ]+217. Found, \%: C 55,28 H 5,07 N $9.96(1 \mathrm{H}$, b.c., $-\mathrm{NH}), 7,80\left(2 \mathrm{H}\right.$, d., O-Ar- $\quad 25,80$. Calculated for $\mathrm{C}_{10} \mathrm{H}_{11} \mathrm{~N}_{3} \mathrm{O}, \%$ : C $\mathrm{H} \mathrm{J}=8,0), 7,2(2 \mathrm{H}, \mathrm{dd}, \mathrm{J}=8,0 \mathrm{~m}-\mathrm{Ar}-\quad 55,29 ; \mathrm{H} 5,06 \mathrm{~N} 25,81$; O 7,37

H), 7,04 (2H, dd, J = 8,0, p-Ar -H), 6,76

1. Tome A. C. Five-Membered Hetarenes with Three or More Heteroatoms. Science of Synthesis. 2004; 13:415-602. [Google Scholar].

2. Alvarez R., Velazquez S., Felix A., Aquaro S., Clercq E., Perno C.-F., Karsson A., Balzarini J., Camarasa M.J. 1,2,3-Triazole-[2,5-Bis-O-(tert-butyldimethylsilyl)-. beta.-D-ribofuranosyl]-3'-spiro-5"-(4"-amino-1",2"-oxathiole 2",2"-dioxide) (TSAO) Analogs: Synthesis and Anti-HIV-1 Activity. Journal of Medical Chemistry. 1994; 4185(37):4185-4194. doi: 10.1021/jm00050a015. [Google Scholar].

3. Khazhieva I.S., Glukhareva T.V., Morzherin Yu.Yu. XXIII Russian molodezhnaya scientific conference «Problems of theoretical and experimental chemistry». Ekaterinburg, UrFU: 23-26 April 2013:506-507. [Google Scholar]. 
И. С. Хажиева, Т. В. Глухарева,

Ю. Ю. Моржерин

ООО «У-Синтез»

620027 , г. Екатеринбург, ул. Луначарского, 60; тел.: (343) 375-48-18;

E-mail:his_inna@mail.ru

\section{Исследование реакции \\ 4-ацетил-1,2,3-триазол-5-олата натрия с производными гидразина}

1,2,3-Триазолы привлекают внимание своей способностью к раскрытию цикла с образованием а-диазоимина и внутримолекулярным перегруппировкам и трансформациям с образованием различных гетероциклических систем. Ранее нами было изучено взаимодействие 1,2,3-триазолата натрия с гидрохлоридами аминов, приводящее к образованию изомерных 1,2,3-триазолов. В данной работе мы представляем исследование взаимодействия 4-ацетил-1,2,3-триазол-5-олата натрия с производными гидразина. Были получены производные 5-метил-1,2,3-триазол-4-фенилкарбоксамидов и бис-фениламид 5,5'-диметил-[1,1']би[[1,2,3]триазолил]-4,4'-дикарбоновой кислоты.

(с) Хажиева И. С., Глухарева Т. В., Моржерин Ю. Ю., 2015

\section{Введение}

Среди производных 1,2,3-триазола обнаружены вещества, обладающие различными видами биологической активности: бактерицидной, противоопухолевой, фунгицидной [1], иммуноподавляющей [2], а также разнообразными техническими свойствами. В последнее время активно продолжается поиск новых производных 1,2,3-триазола, обладающих биологической активностью, и, как следствие этого, наблюдается рост количества публикаций, посвященных 1,2,3-триазолам.
Кроме того, 1,2,3-триазолы проявляют интересные химические свойства, такие как кольчато-цепная изомерия, перегруппировки и трансформации цикла. Это обусловенно способностью 1,2,3-триазолов к раскрытию кольца с образованием $\alpha$-диазоимина.

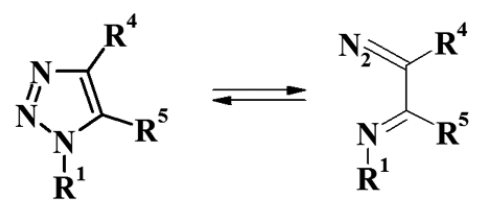


Ранее нами было показано, что взаимодействие 4-ацетил-1,2,3-триазолата натрия 1 с гидрохлоридами аминов протекает с образованием 5-метил1,2,3-триазол-4-карбоксамидов $2^{3}$.

В данной работе было исследовано взаимодействие 4-ацетил-1,2,3-триазо- лата натрия 1 с производными гидразина.

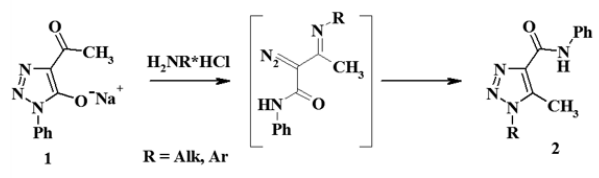

\section{Результаты и обсуждение}

Было показано, что реакция 1,2,3-триазолата натрия 1 с эквимолярным количеством гидрохлоридов гидразинов 3 а-г приводит к 1-амино5-метил-1,2,3-триазол-4-ил-карбоксамидам 4 а-г.

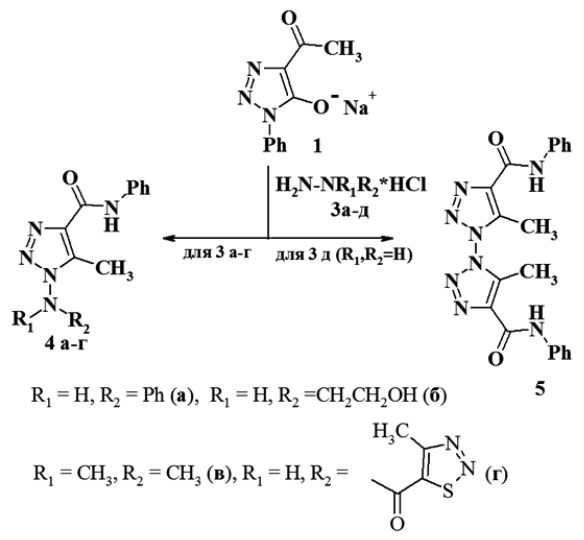

Однако при использовании незамещенного гидразина гидрохлорида в результате реакции был выделен бистриазол 5. В данном случае одна молекула гидразина взаимодействует с двумя молекулами триазолата, что сопровождается перегруппировкой двух триазольных циклов. Возможно такое протекание реакции связано с медленным растворением гидразина гидрохлорида в этаноле.

1-Амино-1,2,3-триазол 6 удалось получить при взаимодействии с гидразингидратом 2-диазоацетоацетанилида 7, полученного подкислением водного раствора 1,2,3-триазолата натрия.

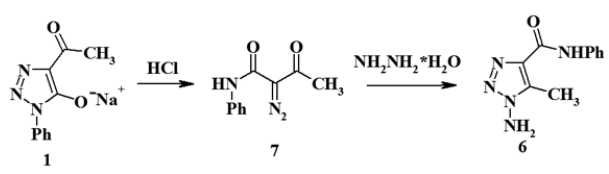

Таким образом, нами были получены неописанные ранее производные фениламидов 5-метил-1-(R-амино)$1 H$ - $[1,2,3]$ триазол-4-карбоновой кислоты и бис-фениламид 5,5'-диметил-[1,1']би[[1,2,3]триазолил]-4,4'дикарбоновой кислоты.

\section{Экспериментальная часть}

Контроль за ходом реакции и индивидуальностью синтезированных соединений проводили методом ТСX на пластинках SolufolUV 254 в системе: хлороформ (проявление УФ лампой). ИК-спектры записаны на спектрофотометре Bruker Alpha. Спектры ЯMР ${ }^{1} \mathrm{H}$ и ${ }^{13} \mathrm{C}$ записаны на спектрометре Bruker
AvanceII (400 и 100 Мгц соотвественно), внутренний стандарт - ТМС, в Лаборатории комплексных исследований и экспертоной оценки органических материалов при ЦКП УрФУ. Массспектры зарегистрированы на спектрометре МАТ 11 (ЭУ, 70 эВ). Температуры плавления определены на приборе 
StuartSMP3 и не исправлены. Элементный анализ выполнен на CHNS-анализаторе PE 2400 SeriesII.

Методика получения 4 а-г и 5

К спиртовому раствору триазолата натрия 1 0,29 г (1,3 ммоль) добавляют
(1,43 ммоль ) гидрохлорида гидразина 3 а-д. Реакционную массу кипятят в течение суток. Охлаждают, выпавший осадок отфильтровывают, промывают этанолом и сушат. Прохождение реакции отслеживают методом ТСХ.

\section{Фениламид 5-метил-1-фениламино-1H-[1,2,3]триазол-4-карбоновой кислоты (4a)}

Осадок белый кристаллический 0,36 г (94,74 \%). $\mathrm{T}_{\text {пл }}=147,3{ }^{\circ} \mathrm{C}$. Спектр ЯMP ${ }^{1} \mathrm{H}:\left(\mathrm{DMSO}-\mathrm{D}_{6}, \mathrm{~d}\right.$, м.д., $\left.J / Г ц\right)$ : $10,41(1 \mathrm{H}, \mathrm{c},-\mathrm{NH}), 10,39(1 \mathrm{H}, \mathrm{c},-\mathrm{NH})$, 7,85(2Н, д, J=8,4, o-Ar-H), 7,33 (2Н, д.д., $J=7,5 ; J=8,4, \mu$-Ar-H), 7,23 (2Н, д.д., $J=7,5 ; J=8,4, \mu-\mathrm{Ar}-\mathrm{H}), 7,09$ (1Н, д.д., $J=8,4 ; J=7,5, p-\mathrm{Ar}-\mathrm{H}), 6,92$ (1Н, д.д.,
$\left.J^{1}=8,4 ; J^{2}=7,5, p-\mathrm{Ar}-\mathrm{H}\right), 6,51(2 \mathrm{H}$, д, $J=8,4, o-\mathrm{Ar}-\mathrm{H}), 2,47\left(3 \mathrm{H}, \mathrm{c}, \mathrm{CH}_{3}\right)$. Спектр ЯMP ${ }^{13} \mathrm{C}$ : (DMSO-D, d, м.д.): $159,61,146,76,139,02,138,59,137,67$, $124,16,121,82,120,88,113,37,8,7$. Найдено, \%: С 65,50, Н 5,17, N 23,91. Вычислено для $\mathrm{C}_{16} \mathrm{H}_{15} \mathrm{~N}_{5} \mathrm{O}, \%$ : C $65,52, \mathrm{H}$ $5,15, \mathrm{~N} 23,88, \mathrm{O} 5,45$.

\section{Фениламид 5-метил-1-(2-гидрокси-этиламино)-1Н-[1,2,3]триазол-4- карбоновой кислоты (46)}

Темно-желтое масло 0,36г (89,45\%). Спектр ЯMP ${ }^{1} \mathrm{H}$ : (DMSO-D, $\mathrm{d}$, м.д.,

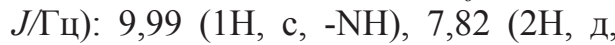
$J=7,6$ o-Ar-H), 7,31 (2Н, д.д., $J=7,6$; $J=8,3, \mu$-Ar-H), 7,20 (1H, т., $J=5,4$, -NH), 7,05 (1Н, д.д., $J=7,4 ; J=7,4, p$-Ar-
Н), 4,06 (1Н, уш.с., -ОН ) 3,56 (2Н, уш.т., $\left.J=5,5,-\mathrm{CH}_{2}\right), 3,26$ (1Н, д.д., $J=5,5$, $\left.J=5,4,-\mathrm{CH}_{2}\right) 2,56\left(3 \mathrm{H}, \mathrm{c}, \mathrm{CH}_{3}\right)$. Найдено, \%: С 55,15 Н 5,80 N 26,80. Вычислено для $\mathrm{C}_{12} \mathrm{H}_{15} \mathrm{~N}_{5} \mathrm{O}_{2}, \%$ : C 55,16, H 5,79, N 26,80, O 12,25.

\section{Фениламид 5-метил-1-диметиламино-1Н-[1,2,3]триазол-4-карбоновой кислоты (4в)}

Темно-желтое масло 0,34 г (92,73\%). Спектр ЯМР ${ }^{1} \mathrm{H}:\left(\mathrm{DMSO}-\mathrm{D}_{6}\right.$, d, м.д., Ј/Гц): 10,06 (1H, с, -NH), 7,82 $(2 \mathrm{H}$, д, $J=7,6, o-\mathrm{Ar}-\mathrm{H}), 7,29$ (2Н, д.д., $J=7,6 ; J=7,4, \mu$-Ar-H), 7,05 (1Н, д.д.,
$J=7,4 ; J=7,4, p$-Ar-H), 3,02 (3H, c, $\left.\mathrm{CH}_{3}\right) 3,06\left(3 \mathrm{H}, \mathrm{c}, \mathrm{CH}_{3}\right) 2,54\left(3 \mathrm{H}, \mathrm{c}, \mathrm{CH}_{3}\right)$. Найдено, \%: С 55,77 Н 6,15 N 28,57. Вычислено для $\mathrm{C}_{12} \mathrm{H}_{15} \mathrm{~N}_{5} \mathrm{O}, \%$ : C 58,76, Н 6,16, N 28,55, О 6,52\%.

\section{(5-метил-4-фенилкарбоксамоил--[1,2,3]триазол-1-ил)-амид 4-метил-[1,2,3]тиадиазол-5-карбоновой кислоты (4г)}

Осадок белый кристаллический 0,52 г $(82,35 \%) . T_{\text {пл }}=175,6^{\circ} \mathrm{C}$. Спектр ЯMP ${ }^{1} \mathrm{H}:$ (DMSO-D, d, м.д., Ј/Гц): $13,23(1 \mathrm{H}, \mathrm{c},-\mathrm{NH}), 10,28(1 \mathrm{H}, \mathrm{c},-\mathrm{NH})$, $7,84(2 \mathrm{H}$, д, $J=7,8, o-A r-H), 7,30(2 \mathrm{H}$, д.д., $J=7,8 ; J=7,9, \mu-\mathrm{Ar}-\mathrm{H}), 7,07$ (1H, т., $J=7,9 ; J=7,9, p$-Ar-H), 2,96 (3H, c, $\left.\mathrm{CH}_{3}\right)$ 2,55 (3H, c, $\left.\mathrm{CH}_{3}\right)$. Найдено, \%: C 48,98 H 3,81 N 28,53. Вычислено для $\mathrm{C}_{14} \mathrm{H}_{13} \mathrm{~N}_{7} \mathrm{O}_{2} \mathrm{~S}, \%$ : C 48,97; $\mathrm{H} \mathrm{3,82,} \mathrm{N}$ 28,55; O 9,32, S 9,34. 


\section{Бис-фениламид 5,5'-диметил-[1,1']6и[[1,2,3]триазолил]-4,4' - дикарбоновой кислоты (5)}

Осадок белый кристаллический 0,30 г $(84,35 \%) . T_{\text {пл }}=217{ }^{\circ} \mathrm{C}$ (лет $\left.180{ }^{\circ} \mathrm{C}\right)$. Спектр ЯМР ${ }^{1} \mathrm{H}:\left(\mathrm{DMSO}^{-\mathrm{D}_{6}}\right.$,

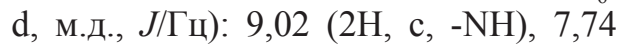
$(4 \mathrm{H}$, д, о-Ar-H $J=8,0), 7,44$ (4H, д.д. , $J=7,6 ; J=8,4$ м-Ar-H), 7,23 (2Н, д.д., $J=7,2, p$-Ar-H) ИК спектр, $\mathrm{v}$, см$^{-1}$ 3308,98 (-NH), 1666,04 (C=O) Maccспектр (ЭУ, 70 эВ), m/z (Іотн (\%)): $[\mathrm{M}]+402(9,14)$. Найдено, \%: С 59,71 Н
$4,47 \mathrm{~N} 18,04$. Вычислено для $\mathrm{C}_{20} \mathrm{H}_{8} \mathrm{~N}_{8} \mathrm{O}_{2}$, \%: C 59,70; H 4,48 N 27,86; O 7,96.

Методика получения 6

К спиртовому раствору 2-диазоацетанилида 7 0,26 г (1,3 ммоль) добавляют 0,07 г (1,43 ммоль) гидразин гидрата. Реакционную массу кипятят в течение суток. Охлаждают, выпавший осадок отфильтровывают, промывают этанолом и сушат. Прохождение реакции отслеживают методами ТCX.

\section{Фениламид 5-метил-1-амино-1H-[1,2,3]триазол-4-карбоновой кислоты}

\section{(4в)}

Осадок белый кристаллический $p$-Ar-H), 6,76 (2H, c., -NH2), 2,54 (1H, 0,32 г $(83,39 \%) . T_{\text {пл }}=199{ }^{\circ} \mathrm{C}$ (лет с, -3). Масс-спектр (ЭУ, 70 эВ), $\mathrm{m} / \mathrm{z}$ $\left.120^{\circ} \mathrm{C}\right)$. Спектр ЯMP ${ }^{1} \mathrm{H}$ : (DMSO-D, d, $\quad$ (Іотн (\%)): [M]+217. Найдено, \%: м.д., J/Гц): 9,96 (1Н, уш.с., -NH), 7,80 С 55,28 Н 5,07 N 25,80. Вычислено для ( $2 \mathrm{H}$, д., о- $\mathrm{Ar}-\mathrm{H} J=8,0), 7,2$ (2Н, д.д., $\quad \mathrm{C}_{10} \mathrm{H}_{11} \mathrm{~N}_{3} \mathrm{O}, \%$ : C 55,29; $\mathrm{H} 5,06 \mathrm{~N} 25,81$; $J=8,0$ м-Ar-H), 7,04 (2Н, д.д., $J=8,0$, O 7,37.

1. Tome A. C. // Five-Membered Hetarenes with Three or More Heteroatoms // Science of Synthesis. 2004 V. 13. New York. P. 415-602.

2. Alvarez R., Velazquez S., Felix A. S., Aquaro S., De Clercq E., Perno C.-F. Karlsson A., Balzarini J. and Camarasa M. J. // Journal of Medicinal Chemistry. 1994. № 37. P. 4185-4194.

3. Хажиева И. С., Глухарева Т. В., Моржерин Ю. Ю. // ХХІІІ Рос. молодеж. науч. конф. «Проблемы теоретической и экспериментальной химии», Екатеринбург, 23-26 апреля 2013 г. : сб. тезисов докладов. Екатеринбург: Урал. ун-т, 2013. C. 506-507. 